\title{
La pluralité des activités, une forme de réactivité commerciale
}

L'exemple de Thomas de Bondié, bourgeois et marchand de Bordeaux (1505-1506)

Pluriactivity and commercial responsiveness: the case of Thomas de Bondié, burgher and merchant of Bordeaux (1505-1506)

\section{Michel Bochaca}

\section{(2) OpenEdition}

Journals

\section{Édition électronique}

URL : http://journals.openedition.org/abpo/2619

DOI : $10.4000 /$ abpo. 2619

ISBN : 978-2-7535-2843-7

ISSN : 2108-6443

\section{Éditeur}

Presses universitaires de Rennes

\section{Édition imprimée}

Date de publication : 30 juin 2013

Pagination : 79-96

ISBN : 978-2-7535-2838-3

ISSN : 0399-0826

Référence électronique

Michel Bochaca, "La pluralité des activités, une forme de réactivité commerciale », Annales de Bretagne et des Pays de l'Ouest [En ligne], 120-2 | 2013, mis en ligne le 30 juin 2015, consulté le 19 avril 2019. URL : http://journals.openedition.org/abpo/2619; DOI : 10.4000/abpo.2619 


\title{
La pluralité des activités, une forme de réactivité commerciale
}

\author{
L'exemple de Thomas de Bondié, bourgeois \\ et marchand de Bordeaux (1505-1506)*
}

\author{
Michel BOCHACA \\ UMR 7266 LIENSs CNRS-Université de La Rochelle
}

Un certain nombre d'affaires traitées entre avril 1505 et décembre 1506 par Thomas de Bondié, marchand et bourgeois de Bordeaux, sont connues grâce à deux registres particuliers instrumentés par le notaire bordelais Jacques Turpaud et conservés à la fin de deux minutiers couvrant respectivement les années 1505-1506 et 1506-1507¹ . Ces actes, du fait de leur nombre (115) et de leur chronologie (20 mois consécutifs entre le 11 avril 1505 et le 9 décembre 1506), éclairent une séquence courte mais cohérente de l'activité commerciale d'un marchand de Bordeaux, ce qui, en l'absence de papiers privés et de comptabilités marchandes conservés pour les XIV et $\mathrm{XV}^{\mathrm{e}}$ siècles, est un élément assez rare pour être souligné ${ }^{2}$.

* La présente étude s'inscrit dans le cadre du projet de recherche Las sociedades urbanas de las ciudades y villas portuarias de la Europa Atlántica en la Baja Edad Media (HAR 2012-31801) dirigé par J. A. Solórzano Telechea et financé par le Ministerio de Economía y Competividad del gobierno de España.

1. Excellent connaisseur des archives notariales bordelaises, Jacques Bernard avait signalé dans sa thèse l'intérêt de ces deux registres particuliers et identifiés Thomas et Louis de Bondié avec le groupe des marchands espagnols qui fréquentèrent Bordeaux après la conquête française où ils incarnaient " un type nouveau d'hommes d'affaires ". Voir BERNARD, Jacques, Navires et gens de mer à Bordeaux (vers 1400-vers 1550), Paris, SEVPEN, 1968, t. 1, p. 25 n. 32 et t. 2, p. 503. FAUMONT, Yohan, Le Commerce du pastel à Bordeaux à travers les registres des actes instrumentés par Jacques Turpaud, notaire, pour Thomas et Louis de Bondié (1505-1506), mémoire de Master inédit, univ. de La Rochelle, 2007. Les deux minutiers de Jacques Turpaud sont conservés aux Archives départementales de Gironde sous les cotes 3 E $12213, \mathrm{f}^{\circ} 329$ à 357 et $3 \mathrm{E} 12214, \mathrm{f}^{\circ} 391$ à $414 \mathrm{v}^{\circ}$. Au début du XVI ${ }^{\mathrm{e}}$ siècle, le style de l'Annonciation était toujours en usage à Bordeaux, notamment chez les notaires. L'année débutait le 25 mars et s'achevait le 24 mars suivant. Toutes les dates ont été restituées en nouveau style.

2. Les plus anciens papiers commerciaux privés conservés pour Bordeaux sont ceux de Fortaney Dupuy, marchand de Saint-Michel et bourgeois de Bordeaux, qui couvrent les 


\section{Thomas de Bondié : un marchand aux activités plurielles}

Mis à part quatre actes qui concernent le frère de Thomas de Bondié, Louis, les 111 actes restants permettent d'identifier 95 transactions distinctes impliquant Thomas de Bondié, dans la mesure où plusieurs d'entre eux se rapportent parfois à une même affaire. Cet échantillon donne un aperçu suggestif des activités économiques auxquelles se livrait Thomas de Bondié car, à l'exception de deux transactions portant sur des maisons, les 93 autres affaires documentées ont toutes un caractère commercial (Annexe 1).

Le pastel figure seul la plupart du temps, parfois associé à d'autres marchandises, dans 55 des 93 affaires commerciales connues. Présent dans 59 \% des transactions, il arrive loin en tête dans une gamme de produits variés dont Thomas de Bondié faisait commerce : vin, blés (froment, avoine), huile d'olive, poisson (hareng, merlus), chanvre et barriques. Cette forte implication dans le commerce du pastel toulousain, dont la production connaît alors son plein développement et qui trouve à Bordeaux une porte de sortie maritime vers les centres drapiers anglais et flamands, constitue la caractéristique majeure des activités économiques de Thomas de Bondié. Elle le distingue des autres marchands bordelais qui opèrent à la même époque et dont les activités sont davantage tournées vers les exportations de vins, l'importation de draps et leur redistribution locale conjointement avec le commerce de détail des grains, du vin, des barriques, de produits finis divers, le tout associé aux prêts d'argent ${ }^{3}$. Nécessitant d'importants moyens financiers, le commerce du pastel a été accaparé, dès sa phase d'essor dans les années 1480-1490, par des marchands castillans, souvent originaires de Burgos. Après s'être déplacés en personne pour la conduite directe de leurs affaires entre Burgos, Toulouse, Bordeaux et Londres, à l'exemple de Diego de Castro dit " de Londres " à la fin du $\mathrm{XV}^{\mathrm{e}}$ siècle $^{4}$, les principaux acteurs tendent à se fixer au début du XVI ${ }^{\mathrm{e}}$ siècle et à contrôler les opérations à partir des points extrêmes du circuit : Toulouse où se concentre et se négocie la production de pastel, et, à l'autre

années 1505-1523. Encore s'agit-il d'un exemple isolé. Voir Mouthon, Fabrice, "Fortaney Dupuy, un marchand bordelais au XvI e siècle ", Annales du Midi, t. 106, 1994, p. 353-373. BochacA, Michel et Micheau, Jacques, Fortaney Dupuy, un marchand de Bordeaux à l'aube de la Renaissance (sous presse).

3. Cf. Fig. 1.a - Le système bordelais. BocHACA, Michel, Les Marchands bordelais au temps de Louis XI. Espaces et réseaux de relations économiques, Bordeaux, Ausonius, 1998, p. 15-56. Reposant sur des produits distincts et fonctionnant selon des logiques commerciales différentes, les circuits d'échanges contrôlés par les marchands bordelais restaient relativement à l'écart des circuits aux mains des marchands castillans avec lesquels ils n'interagissaient guère.

4. BOCHACA, Michel, " Burdeos centro de exportación del pastel de Languedoc hacia Inglaterra y Flandes : el papel de los Castellanos en la organisación de circuitos económicos nuevos al final del siglo XV", dans Entre el Mediterráneo y el Atlántico : España en la formación de un espacio económico europeo (siglos XV-XVIII) - VII ${ }^{\circ}$ congreso de la Asociación española de historia económica, Santiago de Compostela - La Coruña - Vigo (del 13 al 16 de septiembre de 2005). 
bout de la chaine, Londres et Arnemuiden, principaux ports d'arrivée en Angleterre et en Zélande, à partir desquels le pastel est redirigé ensuite vers les centres drapiers anglais, flamands et brabançons. À Toulouse, opèrent des marchands locaux tels Denis de Belbesère, Étienne Ulmyère et Guirauld Ébrard, mais aussi des hommes nouveaux comme Jean de Bernuy, dont la famille est originaire de Burgos ${ }^{5}$. Les destinataires du pastel avec lesquels Thomas de Bondié travaille régulièrement en 1505 et 1506 sont eux aussi des Castillans, à l'exemple de Fernando Dassé et Nicolas de Victory à Londres, ainsi que Coppin et Francisco de Valladolid à Arnemuiden ${ }^{6}$.

Dans ces circuits internationaux du pastel, Bordeaux joue le rôle d'un centre relais ${ }^{7}$. Les balles de pastel descendues par la Garonne sur des embarcations fluviales y sont entreposées avant d'être chargées sur des navires de mer en partance pour l'Angleterre et la Zélande, secondairement la péninsule ibérique (Bilbao, Laredo). Il est possible que Thomas de Bondié ait fait office de consignataire et se soit chargé de réceptionner le pastel à Bordeaux. L'acheminement entre Bordeaux et Toulouse et le stockage à Bordeaux nous échappent faute d'avoir laissé des traces dans les registres de Jacques Turpaud comme dans ceux conservés pour les autres notaires opérant sur place. L'activité principale de Thomas de Bondié consistait à affréter les navires pour le compte des marchands de pastel toulousains et à veiller à leur chargement ${ }^{8}$. Les 49 chargements de pastel sur un total de 57 affrètements et connaissements montrent qu'il s'agit de l'essentiel de sa tâche. Il devait se mouvoir d'autant plus à son aise dans ces circuits largement dominés par des marchands castillans qu'il appartenait

5. CASTER, Gilles, Le Commerce du pastel et de l'épicerie à Toulouse (1450 environ à 1561), Toulouse, Privat, 1962. CASADO Alonso, Hilario, " Le rôle des marchands castillans dans la commercialisation internationale du pastel toulousain (XV et XVI ${ }^{\mathrm{e}}$ siècles) ", dans CARDON, Dominique et HANsJürgen, Müllerott (dir.), Actes du $2^{\mathrm{e}}$ Congrès international Pastel, indigo et autres plantes tinctoriales : Passé, présent, avenir (Toulouse, 8-10 juin 1995), Arnstadt, Thüringer Chronik-Verlag, 1998, p. 65-70. CASADO Alonso, Hilario, El Triunfo de Mercurio. La presencia castellana en Europa (siglos XV y XVI), Burgos, Cajacírculo, 2003. CASADO AlONSO, "Las redes comerciales castellanas en Europa (siglos XV y XVI) ", dans Casado Alonso, Hilario et García Baquero, Antonio (dir.), Comercio y hombres de negocios en Castilla y Europa en tiempos de Isabel la Católica, Madrid, Sociedad Estatal de culturales Conmemoraciones, 2007. CASADO ALONSO, Hilario, "Crecimiento économico, redes de comercio y de fiscalidad en Castilla a fines de la Edad Media ", dans BonACHIA HERnANDO, Juan Antonio et CARVAJAL DE LA VEGA, David (dir.), Los negocios del hombre. Comercio y rentas en Castilla. Siglos XV y XVI, Valladolid, Castilla Ediciones, 2012, p. 17-36. CASADO AlONSO, Hilario, "Los agentes castellanos en los puertos atlánticos : los ejemplos de Burdeos y de los Países Bajos (siglos XV y XVI) ", dans FÁBREGAS GARCíA, Adela (dir.), Navegación y puertos en época medieval y moderna, Grenada, La Nao, 2012, p. 163-194 (171-180).

6. Les membres de la famille des Valladolid établis à Middelburg ont été présentés par FAGEL, Raymond, De Hispano-Vlaamse Wereld. De contacten tussen Spanjaarden en Nederlanders, 1496-1555, Bruxelles, 1996, p. 114-117. Renseignement bibliographique aimablement communiqué par Hilario Casado Alonso (Université de Valladolid).

7. Cf. Fig. 1.b - Le système castillan et Fig. 2 - Les circuits du pastel de Jean Bernuy.

8. Arch. dép. de Gironde, 3 E 12213, f $\mathrm{f}^{\circ} 335,5$ juin 1505. Thomas de Bondié inspecte luimême le Christophe de Bordeaux avant de convenir avec le maître que le navire ne peut pas porter plus de 387 balles de pastel. 
lui-même à cette communauté. Membre de la famille des Buendia, dont le patronyme a été déformé en Bondié par les scribes gascons ${ }^{9}$, il est d'abord associé à son beau-père, Pierre del Poyo, auquel il succède en 1505 probablement après la mort de ce dernier. Marié à Isabelle del Poyo, il tenait feu vif dans la ville depuis suffisamment de temps pour acquérir le statut de bourgeois de Bordeaux et jouir des privilèges commerciaux et fiscaux qui y étaient attachés.

Si Thomas de Bondié agit avant tout pour le compte d'autres marchands, les rapports directs qu'il entretient avec les maîtres de navire faisant en escale à Bordeaux lui permettent d'obtenir de ces derniers des facilités et des rabais dont il profite pour développer de façon parallèle ses propres affaires. Il n'est pas rare de le voir embarquer quelques barriques de vin ou balles de pastel dont il a la propriété aux côtés de celles des marchands toulousains pour le compte desquels il agit ${ }^{10}$. Ces marchandises personnelles sont chargées " libres de fret ", c'est-à-dire sans payer le coût du transport, ce qui lui permet d'augmenter ses marges bénéficiaires ${ }^{11}$. Entre le 25 avril 1505 et le 11 avril 1506, Thomas de Bondié procède à 13 expéditions quittes de fret pour un total de 72 balles de pastel et de 5 tonneaux et une barrique de vin, auxquels il faut ajouter 14 pièces de cuir, deux douzaines et demie de planches de chêne et une demi-barrique de vinaigre. La possibilité de charger gratuitement pour son compte offrait indiscutablement des avantages, d'autant que son statut de bourgeois de Bordeaux l'exonérait totalement ou en partie du paiement des droits de douane, ou coutumes, pour les marchandises dont il était propriétaire.

Au premier abord, les actes dont nous disposons montrent Thomas de Bondié agissant à la manière d'un consignataire. Opérant pour le compte de tiers, on peut supposer qu'il réceptionnait à Bordeaux le pastel expédié depuis Toulouse. Nous le voyons surtout rediriger ce pastel vers d'autres correspondants établis à Londres ou à Arnemuiden qui lui étaient sans doute désignés par ses donneurs d'ordres toulousains. On ignore comment il était

9. Nous remercions Hilario Casado Alonso d'avoir attiré notre attention sur ce rapprochement.

10. Arch. dép. de Gironde, 3 E 12213, f ${ }^{\circ} 331,25$ avril 1505 (18 balles de pastel et 2 tonneaux de vin); $\mathrm{f}^{\circ} 342,5$ novembre 1505 (4 balles de pastel); $\mathrm{f}^{\circ} 344 \mathrm{v}^{\circ}, 8$ novembre 1505 (10 balles de pastel); $\mathrm{f}^{\circ} 345 \mathrm{v}^{\circ}, 10$ novembre 1505 (10 balles de pastel); $\mathrm{f}^{\circ} 346,11$ novembre 1505 (20 balles de pastel et un tonneau de vin); $\mathrm{f}^{\circ} 346 \mathrm{v}^{\circ}-347,3$ décembre 1505 (10 balles de pastel); $\mathrm{f}^{\circ} 347-347 \mathrm{v}^{\circ}, 4$ décembre 1505 (un tonneau de vin); $\mathrm{f}^{\circ} 356-356 \mathrm{v}^{\circ}, 5$ mars 1506 (2 barriques de vin) ; f ${ }^{\circ} 356-356 \mathrm{v}^{\circ}, 20$ mars 1506 (une barrique de vin). Ibid., $3 \mathrm{E} 12214$, $\mathrm{f}^{\circ} 391-391 \mathrm{v}^{\circ}, 30$ mars 1506 (une barrique de vin); $\mathrm{f}^{\circ} 392 \mathrm{v}^{\circ}-393,11$ avril 1506 (une barrique de vin); $\mathrm{f}^{\circ} 393 \mathrm{v}^{\circ}, 21$ avril 1506 (14 pièces de cuir) ; f $396-396 \mathrm{v}^{\circ}, 12$ mai 1506 (2 douzaines et demie de planches de chêne et une demi-barrique de vinaigre).

11. BochacA, Michel et Tranchant, Mathias, "Du golfe de Gascogne à la Picardie et à la Flandre maritime : le déplacement par mer des hommes et des marchandises à la fin du $\mathrm{XV}^{\mathrm{e}}$ siècle ", dans Se déplacer du Moyen Âge à nos jours, Actes du $6^{\mathrm{e}}$ colloque européen de Calais (2006-2007), Calais, Les Amis du Vieux Calais, 2009, p. 135-146. Dans les années 1470-1480, le coût moyen du transport d'un tonneau de vin entre Bordeaux et Calais représentait environ le quart de la valeur marchande de celui-ci. 
rémunéré pour ce travail (système de commissions?). Son rôle était celui d'un intermédiaire au service d'autres marchands, dont certains, à l'exemple des marchands de pastel toulousains, avaient une envergure économique bien supérieure à la sienne ${ }^{12}$. Signe de la modestie de ses moyens, il réalise seulement deux chargements pour son propre compte, dont un portant sur quatre barils d'huile ${ }^{13}$. S'il charge à son nom 32 tonneaux et demi de vin et 68 petites balles de pastel à destination de Laredo, rien n'interdit de penser que ces marchandises allaient " aux aventures et périls " d'un autre marchand qui endossait le risque en cas de perte du navire. Pour autant, Thomas de Bondié ne se cantonne pas au rôle d'un simple consignataire. Comme pour les expéditions de pastel, dont il profite pour faire ses propres envois, il tire partie de la dynamique générale des affaires et des réseaux commerciaux dans lesquels il évolue pour avancer ses pions. Au gré des opportunités, il vend du vin ${ }^{14}$ et du pastel ${ }^{15}$, il prête de l'argent ${ }^{16}$, il achète en gros des barriques neuves à des tonneliers bordelais ${ }^{17}$ pour les revendre ensuite en petites quantités à des particuliers ${ }^{18}$. On devine cependant au détour de certains actes qu'une partie des activités de Thomas de Bondié nous échappe.

12. Outre les chargements de pastel opérés pour le compte de Toulousains, Thomas de Bondié leur sert régulièrement de relais. Le 27 octobre 1505, il reçoit quittance d'Étienne du Montelle, serviteur de Gloussé de Cibile, marchand de Rouen, auquel Jean de Bernuy, marchand de Toulouse, devait 1260 l. 10 s. t. (Arch. dép. de Gironde, 3 E 12213, f ${ }^{\circ} 339$ ). Le 24 décembre 1505, il reconnaît avoir reçu de Coppin de Valladolid, marchand et bourgeois de Middelburg, 11 lasts de harengs qu'il a vendus. Avec cet argent, il a acheté 15 tonneaux de vin et 16 balles de pastel qui ont été chargés sur la Barbe de Penmarch (Ibid., 3 E 12213, f ${ }^{\circ}$ 349-350). Trois actes datés de juillet 1506 le montrent comme le correspondant à Bordeaux d'un trafic de blé à destination du Portugal organisé par Pierre Lopes et Diego de Castro (Ibid., 3 E 12214, $\mathrm{f}^{\circ}$ 405, 7 juillet 1506 ; f ${ }^{\circ} 406-406 \mathrm{v}^{\circ}$, 7 juillet 1505 et $\mathrm{f}^{\circ} 406 \mathrm{v}^{\circ}-407 \mathrm{v}^{\circ}, 17$ juillet 1506).

13. Ibid., 3 E 12213 , f ${ }^{\circ} 350-350 \mathrm{v}^{\circ}, 26$ décembre 1505 , connaissement pour 4 barils d'huile d'olive expédiés à Londres, le fret restant à fixer; 3 E 12214, f ${ }^{\circ} 21$, avril 1506, connaissement pour 32,5 tonneaux de vin et 68 petites balles de pastel à destination de Laredo pour le prix de 40 sous tournois le tonneau de fret, plus 14 pièces de cuir quittes de fret.

14. Ibid., 3 E 12213, $\mathrm{f}^{\circ} 350,24$ décembre 1505, reconnaissance de dette de Jean Le Couleur, maître de la Pentecoste de Penmarch, envers Thomas de Bondié pour l'achat de 2 tonneaux de vin qu'il reconnaît avoir goûtés.

15. Ibid., $3 \mathrm{E} 12213$, $\mathrm{f}^{\circ} 351 \mathrm{v}^{\circ}$ et $\mathrm{f}^{\circ} 351 \mathrm{v}^{\circ}-352,9$ février 1506 , reconnaissance de dette de Guilhem de Lange et de Jehan de Pey de Troy, marchands de Bordeaux, envers Thomas de Bondié pour l'achat de 5 pipes de pastel. Thomas de Bondié se porte garant de l'aloi des 5 pipes de pastel.

16. Ibid., 3 E 12214, $\mathrm{f}^{\circ} 399 \mathrm{v}^{\circ}, 26$ mai 1506, reconnaissance de dette de Jehan de Bernerichs, paroissien de Sainte-Eulalie (Bordeaux), envers Thomas de Bondié pour un prêt de 50 francs bordelais remboursable à la Saint Michel suivante. Acte cancellé le 29 octobre 1506 .

17. Ibid., 3 E 12213 , f 333 v $^{\circ}, 7$ mai 1505. Ibid., 3 E 12214, $\mathrm{f}^{\circ} 394,21$ avril 1506 ; $\mathrm{f}^{\circ} 394 \mathrm{v}^{\circ}$, 21 avril $1506 ; \mathrm{f}^{\circ} 394 \mathrm{v}^{\circ}-395,21$ avril $1506 ; \mathrm{f}^{\circ} 395,21$ avril $1506 ; \mathrm{f}^{\circ} 397 \mathrm{v}^{\circ}-398 ; 11$ mai 1506 ; $\mathrm{f}^{\circ} 398,15$ mai 1506 .

18. Ibid., 3 E 12213 , f 337 v, 9 septembre 1505. Ibid., 3 E 12214 , f ${ }^{\circ} 408$ v $^{\circ}-409$, 11 septembre $1506 ; \mathrm{f}^{\circ} 409,15$ septembre $1506 ; \mathrm{f}^{\circ} 409,15$ septembre $1506 ; \mathrm{f}^{\circ} 409 \mathrm{v}^{\circ}, 15$ septembre $1506 ; \mathrm{f}^{\circ} 409 \mathrm{v}^{\circ}, 16$ septembre $1506 ; \mathrm{f}^{\circ} 409 \mathrm{v}^{\circ}-410,16$ septembre $1506 ; \mathrm{f}^{\circ} 410,5$ septembre $1506 ; \mathrm{f}^{\circ} 410-410 \mathrm{v}^{\circ}, 23$ septembre 1506 . Le rôle central des marchands bordelais dans la redistribution des barriques neuves auprès des particuliers a été récemment mis en 
À deux reprises, le 5 novembre 1505 et le 20 janvier 1506, il a recours à Étienne Ulmyère pour tenter de recouvrer des créances qui lui sont dues dans les pays de la moyenne Garonne, sans que la nature exacte des affaires soit précisée ${ }^{19}$. Le 7 avril 1506, il donne procuration à Jehan Chymenez, marchand de Bordeaux, pour que celui-ci se fasse remettre par Anthony Bonencontre, marchand de Montauban, et Jehan Benaguet, marchand de Toulouse, l'argent provenant de la vente de neuf et de dix pipes de merlus que Thomas de Bondié leur a respectivement envoyées pour les écouler à son profit ${ }^{20}$. Le 15 avril 1506, il donne une autre procuration à Pedro de Saint-Étienne, marchand espagnol demeurant à Toulouse, absent au moment de la passation de l'acte, pour recouvrer 50 l. 9 s. 8 d. t. que lui doit Pedro de Lambiers, marchand navarrais demeurant à La Rochelle ou à La Châtaigneraie en Poitou ${ }^{21}$. S'agit-il de trafics propres à Thomas de Bondié? On peut en douter car, en sens inverse du pastel, il sert de relais à des correspondants étrangers pour écouler à partir de Bordeaux des marchandises importées. Le 24 décembre 1505, Thomas de Bondié reconnaît avoir reçu de Coppin de Valladolid, marchand de Middelburg, 11 lasts de harengs qu'il a vendus. Avec cet argent, il a acheté 15 tonneaux de vin et 16 balles de pastel qui sont chargés sur la Barbe de Penmarch et réexpédiés à son correspondant castillan établi en Zélande ${ }^{22}$. Or ce même Coppin de Valladolid figure parmi les destinataires zélandais du pastel chargé à Bordeaux par les soins de Thomas de Bondié.

\section{Opportunisme commercial et capacité d'adaptation à la conjoncture}

Alors que les expéditions de pastel s'étalent assez largement sur 13 des 20 mois couverts par les deux registres particuliers instrumentés par Jacques Turpaud, l'année 1506 voit un changement notable dans les activités de Thomas de Bondié (Annexe 2). Cinq chargements de blé sont expédiés à destination de la péninsule ibérique : un premier en mars vers SaintSébastien et Pasajes, suivi de quatre autres en juin et juillet vers Lisbonne et Setubal, avec, pour le dernier, la possibilité de poursuivre le voyage jusqu'à Cadix et Sanlucar de Barrameda.

Il faut tout d'abord rappeler que les sorties de blé depuis Bordeaux sont peu fréquentes dans la mesure où la ville doit régulièrement importer des grains de Saintonge et des pays de la moyenne Garonne pour couvrir les besoins de sa population que les campagnes bordelaises ne suffisent pas à nourrir ${ }^{23}$. Les exportations sont soumises à une autorisation préalable

lumière : PORCHER, Kevin, La Tonnellerie bordelaise après la guerre de Cent Ans (vers 1450vers 1480), mémoire de master inédit, univ. de La Rochelle, 2006.

19. Arch. dép. de Gironde, $3 \mathrm{E} 12213$, f ${ }^{\circ} 341 \mathrm{v}^{\circ}-342,5$ novembre 1505 et f f $350 \mathrm{v}^{\circ}, 20$ janvier 1506.

20. Ibid., 3 E $12214, \mathrm{f}^{\circ} 391 \mathrm{v}^{\circ}-392$.

21. Ibid., 3 E $12214, \mathrm{f}^{\circ} 393-393 \mathrm{v}^{\circ}$.

22. Ibid., 3 E $12213, \mathrm{f}^{\circ} 349-350$.

23. Mouthon, Fabrice, Les Blés du Bordelais et du Bazadais. L'économie céréalière dans les diocèses de Bordeaux et de Bazas (vers 1300-vers 1550), thèse d'université inédite, Bordeaux, 1993, t. 1, p. 129-229. 
par les officiers du roi et ne peuvent intervenir qu'en période d'excédent pour Bordeaux et sa région. Le chargement à destination de Saint-Sébastien et de Pasajes n'est pas surprenant outre mesure car le Guipúzcoa et la Biscaye souffraient plus encore que le Bordelais d'une pénurie chronique de grains ${ }^{24}$. D'autre part, les ports cantabriques ne sont pas inconnus de Thomas de Bondié qui expédie du pastel vers Bilbao le 5 juin 1505 et le 12 mai $1506^{25}$, ainsi que du vin, du pastel et du cuir vers Laredo le 21 avril $1506^{26}$. Les envois de blé vers Lisbonne et Setubal sont en revanche inhabituels pour autant qu'on puisse en juger à partir des maigres sources faisant état de relations commerciales entre Bordeaux et le Portugal. Les deux villes portugaises pourvoyaient à l'occasion Bordeaux, la première en sucre de Madère et en épices, la seconde en sel et en sardines salées ${ }^{27}$. Autre élément inaccoutumé dans les affaires traitées par Thomas de Bondié, les quatre envois de blé vers le Portugal donnent lieu à une intense activité qui se manifeste tant par le nombre des actes consignés dans le registre (9 au total) que par leur contenu qui laisse entendre un échange d'informations soutenu entre les acteurs de ce trafic. Tout se passe comme si le réseau commercial auquel appartient Thomas de Bondié s'activait soudainement afin de mobiliser les énergies et les capitaux vers des marchés offrant des perspectives de profit intéressantes mais situés hors de l'aire d'activité habituelle de ses membres. Si nous ignorons la provenance des informations et leur mode de transmission, il ne fait aucun de doute que les hommes d'affaires castillans et leurs associés toulousains dans le sillage desquels se mouvait Thomas de Bondié ont réagi en parfaite connaissance de cause. À partir de 1498, Lisbonne et Setubal servent de plaque tournante pour les relations portugaises avec l'Afrique et connaissent une forte demande en céréales pour soutenir cette expansion maritime. À côté des permis régulièrement concédés par le roi pour importer des blés étrangers, les violences dont les juifs convertis sont victimes à Lisbonne en 1506 sont le signe de graves difficultés d'approvisionnement ${ }^{28}$. La possibilité de poursuivre de Lisbonne vers Cadix et Sanlucar de Barrameda envisagée en juillet 1506 lors de la préparation du dernier voyage connu trouve, elle aussi, une explication dans la crise de subsistance que connaît alors l'Andalousie $^{29}$. C'est donc sur la foi d'informations précises et de haute valeur

24. Arízaga Bolumburu, Beatriz, "El Abastecimiento de las villas vizcaínas medievales ", dans La Ciudad Hispánica ss. XIII-XVI, Madrid, 1985, p. 293-316. AríZAGA BoluMBURU, Beatriz, "La perception de la frontière en Guipúzcoa au Moyen Âge ", dans MEnJot, Denis (dir.) Les Villes frontières (Moyen Âge, Époque Moderne), Paris, l'Harmattan, 1997, p. 129-143.

25. Arch. dép. de Gironde, 3 E $12213, f^{\circ} 335$ et 3 E $12214, f^{\circ} 396-396$ v $^{\circ}$.

26. Ibid., $3 \mathrm{E} 12214, \mathrm{f}^{\circ} 393 \mathrm{v}^{\circ}$.

27. BERnARD, Jacques, Navires et gens de mer..., op. cit., t. 1, p. 216 n. 175.

28. Renseignements aimablement fournis par Amélia Andrade et Cláudia Silveira (Université Nouvelle de Lisbonne).

29. Yun CASAlilla, Bartolome, Crisis de subsistencia y conflictividad social en Córdoba a principios del siglo XVI, Córdoba, Diputación Provincial, 1980. MARTín GuTiÉRrez, Emilio, "La crisis de 1503-1507 en Andalucía. Reflexiones a partir de Jerez de la Frontera ", dans Oliva Herrer, Hipólito Rafael et Benito i Monclús, Pere (dir.), Crisis de subsistencia y crisis 
économique que ces opérations commerciales sortant de l'ordinaire, tant par le produit traité que par les marchés prospectés, sont entreprises. Les nombreux envois de blé effectués en 1506 à partir de Bordeaux à destination du Guipúzcoa, de la Biscaye, du Portugal et de l'Andalousie qui, au-delà de Thomas de Bondié, impliquent d'autres marchands, témoignent de la grave pénurie qui sévissait alors dans la péninsule ibérique ${ }^{30}$.

Les deux connaissements passés le 25 juin 1506 par Étienne Rousseau et Arnaut Cluseau, respectivement maîtres du Martin de Ré et du Pierre de Marennes ${ }^{31}$, ont été précédés les 3 et 9 juin par deux contrats d'affrètement en bonne et due forme grâce auxquels Thomas de Bondié réservait par avance le service des deux navires ${ }^{32}$. L'affrètement de la Jeannette de Crozon sans doute infructueux dans un premier temps, comme en témoigne la minute inachevée et cancellée en date du 23 juin, est finalement conclu deux jours plus $\operatorname{tard}^{33}$. Le Martin et le Pierre doivent " conduire du premier temps convenable [...] au port de Lisbonne ou Setubal en droiture " 187 et 140 pipes de froment. Le maître de la Jeannette s'engage quant à lui charger le plus tôt possible tout le blé que son navire peut emporter. Thomas de Bondié avance aux deux premiers maîtres 14 et 12 écus déductibles du fret, mais qui doivent lui être remboursés si les navires ne parviennent pas à bon port.

On peut raisonnablement penser que le Martin et le Pierre ont emporté leur cargaison de blé vers le Portugal. La chose n'est pas aussi certaine pour la Jeannette car, à partir du début de juillet, la poursuite des opérations ne se déroule pas aussi bien que prévu. L'importance de l'affaire, éminemment spéculative et qui s'apparente à un " gros coup ", peut expliquer la venue à Bordeaux de Louis de Bondié qui, durant cette courte période, agit tantôt seul, tantôt associé à Thomas. Le 7 juillet 1506, il fait dresser un acte par lequel il déclare n'avoir pas reçu la lettre de change que Denis de Belbesère, marchand de Toulouse, lui a envoyée quinze jours plus tôt par le truchement de Jean de Bernuy ${ }^{34}$. Faute de disposer des 1000 livres promises, il informe son correspondant toulousain qu'il a dû laisser appareiller les navires affrétés. La Jeannette figurait-elle au nombre de ces derniers? Il précise qu'il lui faut dès lors procéder à d'autres affrètements et acheter du blé à plus haut prix, autant de contretemps qui ralentissent le cours des opérations et réduisent leur rentabilité. Entre-temps, Salvat de Lahet, serviteur de Jean de Bernuy, a tenté de faire parvenir la somme, comme en témoigne la copie, établie par le notaire Jacques Turpaud, d'une lettre dudit Salvat de Lahet écrite en castillan et donnant l'ordre de payer à Louis

agraria en la Edad Media, Séville, Universidad de Sevilla, 2007, p. 277-302. Références bibliographiques aimablement communiquées par Antonio Collantes de Teran (Université de Séville).

30. Bernard, Jacques, Navires et gens de mer..., op. cit., t. 3, p. 202-242.

31. Arch. dép. de Gironde, 3 E 12214, f $\mathrm{f}^{\circ} 401 \mathrm{v}^{\circ}-402,402 \mathrm{v}^{\circ}-403$.

32. Arch. dép. de Gironde, 3 E $12214, f^{\circ} 399 v^{\circ}-400$ et $400-400 v^{\circ}$.

33. Arch. dép. de Gironde, 3 E $12214, \mathrm{f}^{\circ} 401 \mathrm{v}^{\circ}$ et $403-403 \mathrm{v}^{\circ}$.

34. Arch. dép. de Gironde, 3 E 12214, f ${ }^{\circ} 404$. 
de Bondié 1000 livres dues par Alonce de Corroal ${ }^{35}$. Peine perdue comme l'atteste l'acte du 7 juillet.

La mobilisation du réseau Belbesère-Bernuy ne s'arrête pas là. Outre Louis venu seconder son frère Thomas à Bordeaux, d'autres correspondants s'activent. Le 6 juin et le 13 juin, par deux contrats passés devant un notaire de Bruges, Jean Bisine, marchand de Bordeaux, a vendu à Diego de Castro, marchand espagnol, 800 boisseaux (?) ${ }^{36}$, et à Pierre Lopes, marchand de Calatayud, 2400 boisseaux de blé, pour le prix de 200 écus $^{37}$, à charger dans les deux cas à Bordeaux sur les navires affrétés, 15 jours après leur arrivée. Le 7 juillet, Jean Bisine institue son frère Pierre, marchand de Marmande, garant du chargement du blé et reconnaît avoir reçu de Louis et Thomas de Bondié les 200 écus promis par Pierre Lopes. Le 5 août, Jean Bisine donne quittance à Thomas de Bondié pour le paiement de 100 écus en relation avec un chargement de blé pour Pierre Lopes. Si le blé n'est pas à bord de la Charrette de Penmarch le 12 août, il s'engage à rembourser les 100 écus, ce qu'il n'eut pas à faire car l'acte fut cancellé le 20 août après exécution du contrat ${ }^{38}$. Parallèlement, le 17 juillet, le maître de la Marie du Croisic, dont le navire avait été affrété le 18 juin par Thomas de Bondié, reconnaît avoir chargé à Libourne 83 tonneaux et 8 boisseaux de blé appartenant à Rodrigo de Valladolid ${ }^{39}$. Ce voyage plus tardif vers Lisbonne et Setubal risquant de ne pas être aussi rentable, une solution de rechange est envisagée avec la poursuite vers Cadix et Sanlucar de Barrameda après avoir pris les ordres à Lisbonne. Une option est prise par avance sur le navire pour un possible retour à Bordeaux avec 35 caisses de sucre à son bord.

En raison de la fragilité des grains dont le chargement se faisait en vrac ou dans des sacs, ce qui les exposait à la moisissure tant par l'humidité de l'air que par les infiltrations d'eau dans la coque des navires ou les paquets de mer embarqués par mauvais temps, des garanties particulières, qui ne figurent pas dans les expéditions de pastel et de vin, sont prises pour assurer la bonne conservation de la marchandise pendant le voyage ${ }^{40}$. D'autre part, envoyés vers des destinations méridionales imposant une traversée en droiture du golfe de Gascogne puis une navigation le long des côtes galiciennes et portugaises qui leur étaient moins familières que celles de Bretagne, de Normandie, de Flandre ou du sud de l'Angleterre, il est prévu

35. Arch. dép. de Gironde, 3 E $12214, \mathrm{f}^{\circ} 404 \mathrm{v}^{\circ}$.

36. Arch. dép. de Gironde, 3 E 12214, 406-406 v. L'unité de mesure semble avoir été omise par inadvertance : "le nombre de huyt cens de bledz " (sic). Il pourrait s'agir de boisseaux.

37. Arch. dép. de Gironde, 3 E $12214, \mathrm{f}^{\circ} 405 \mathrm{v}^{\circ}$.

38. Arch. dép. de Gironde, 3 E $12214, \mathrm{f}^{\circ} 408$.

39. Arch. dép. de Gironde, 3 E $12214, \mathrm{f}^{\circ} 406 \mathrm{v}^{\circ}-407 \mathrm{v}^{\circ}$.

40. Arch. dép. de Gironde, 3 E 12214, $\mathrm{f}^{\circ} 403 \mathrm{v}^{\circ}, 25$ juin 1506. «Et doibt ledit maistre auoir sondit nauire bien estanche, garny de gens et d'aultres chouses neccessaires pour la conduyte dudit nauire scelon le voiage ou au present il va, et au cas que par deffault dudit maistre ou nauire ou gens dudit nauire ledit blé feust gasté etc. le doibt reparer. " 
que les deux maîtres de navires saintongeais fassent appel aux services de pilotes et de lamaneurs pour les manœuvres dans le port d'arrivée, toute escale en cours de route leur étant interdite sauf cas de force majeure ${ }^{41}$.

Après une brève et subite embellie documentaire entre mars 1505 et décembre 1506, nous perdons la trace de Thomas de Bondié sans savoir si l'obscurité qui s'abat sur lui au-delà de 1506 est due à la mauvaise conservation des sources, à un décès ou bien à un départ de Bordeaux. Bien que les informations dont nous disposons soient ténues et fragmentaires, elles permettent néanmoins d'entrevoir à partir de Bordeaux le fonctionnement d'un système commercial centré sur le proche Atlantique et déployé à travers l'ouest de l'Europe depuis l'Angleterre et la Zélande au nord, jusqu'à l'Andalousie au sud, en passant par le Portugal et les ports castillans et français du golfe de Gascogne.

Intermédiaire dans un réseau commercial qui l'englobe et le dépasse, Thomas de Bondié n'en constitue pas moins un rouage actif. Tout en se livrant à quelques trafics propres, il agit surtout pour le compte de marchands d'une envergure supérieure à la sienne, qui opèrent à Toulouse (Denis de Belbesère, Étienne Ulmyère, Giraud Ébrard et Jean de Bernuy) ou à Middelburg (Coppin de Valladolid). Son rôle à Bordeaux consiste à affréter des navires pour expédier vers Londres et Arnemuiden le pastel que ses donneurs d'ordres lui envoient depuis Toulouse. Il participe au bon fonctionnement des opérations quitte à recevoir du renfort, à l'exemple de l'intervention de son frère Louis en juin et juillet 1506, lorsque les affaires se complexifient avec les expéditions de blé vers le Portugal et l'Andalousie.

Cette pluralité des activités, même si elle s'exerce de façon occasionnelle, démontre la réactivité d'un groupe de marchands face aux opportunités du marché. Elle témoigne de leur capacité à exploiter rapidement des informations économiques sur l'état des marchés, à réorienter les énergies et les capitaux des membres du réseau vers des produits qui ne sont pas ceux couramment traités. Dans le cas présent, la diversification des activités n'a pas pour objet de prévenir un quelconque risque mais, au contraire, elle s'accompagne d'une prise de risque commercial et financier en lançant hommes, navires et produits sur des itinéraires maritimes nouveaux, à destination de marchés différents de ceux qui étaient habituellement fréquentés.

41. Ibid., $3 \mathrm{E} 12214, \mathrm{f}^{\circ} 403 \mathrm{v}^{\circ}$. " Et sera paié le pilotaige et touaige et lamainage acoustumé et de toutes abaries tant deça que par della [...] Et doibt aller a droicte voye sans sajourner ny aborder a la couste d'Espaigne ainsi que Dieu leur en donnera le temps. " 
Figure 1 -Superposition des circuits commerciaux du vin et du pastel passant par le port de Bordeaux (fin $X V^{e}$-début $X V V^{e}$ siècle)

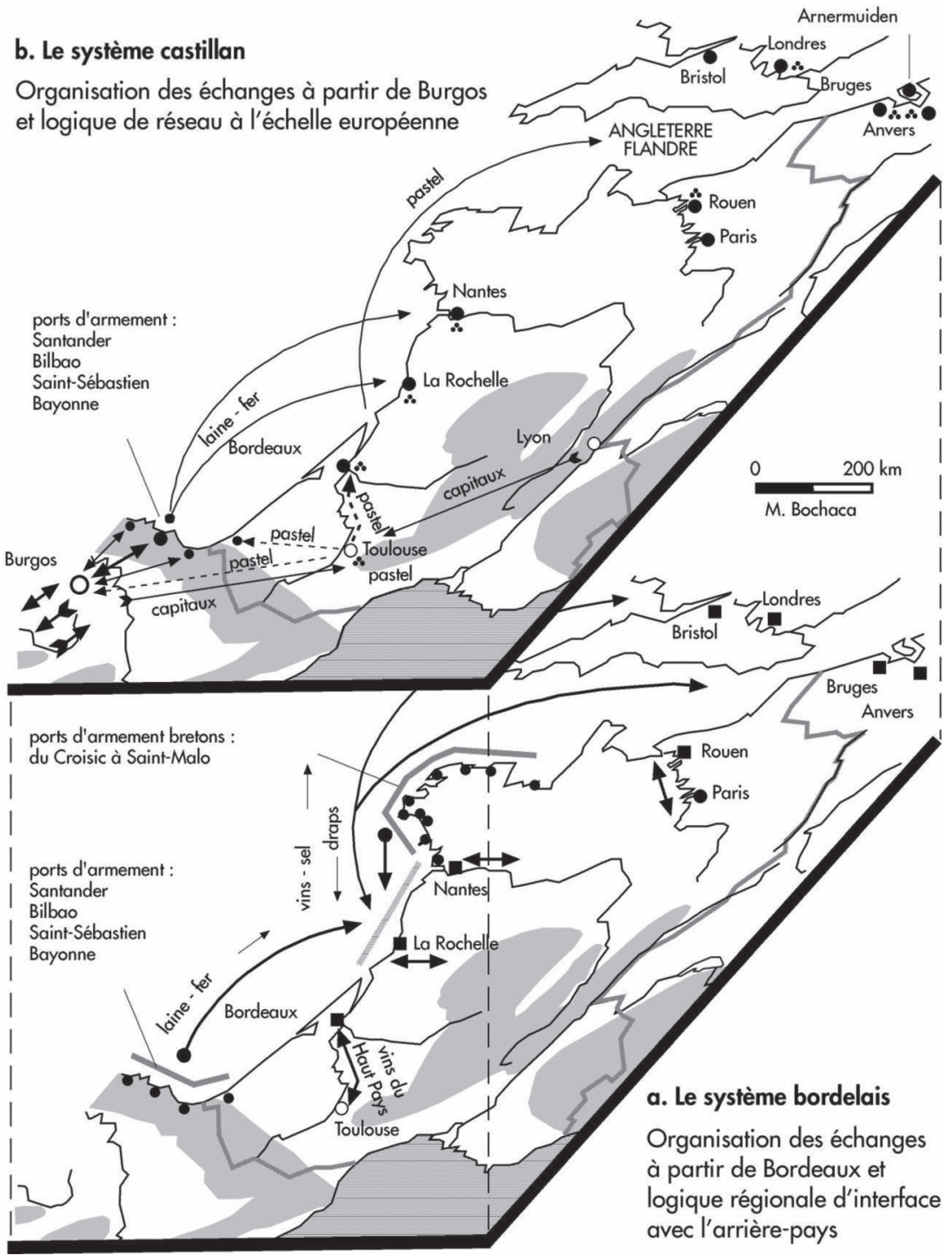


Figure 2 - Les circuits du pastel de Jean Bernuy (1505-1506)

Londres Arnemuiden 4. commis, associés

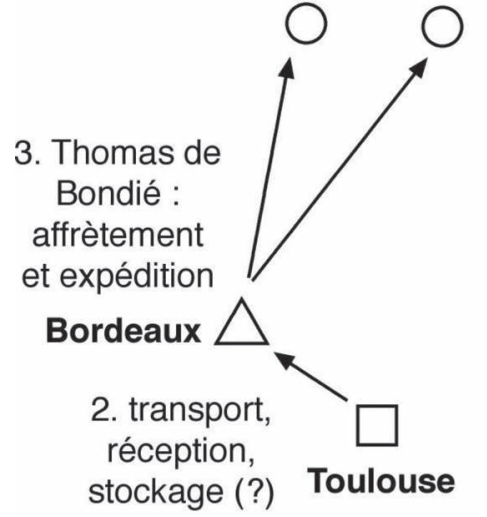

$\square$ centre de production

centre d'exportation castillans : réception

1. Jean de Bernuy:

contrôle de la production

\footnotetext{
centre de consommation ou de redistribution
} 


\section{Annexe 1 - Typologie des actes instrumentés par Jacques Turpaud pour le compte Thomas de Bondié en 1505-1506}

\begin{tabular}{|l|c|}
\hline Chargements de navires : & 57 \\
- connaissements & 51 \\
- affrètements & 6 \\
\hline $\begin{array}{l}\text { Reconnaissances de dette (pour vente } \\
\text { de marchandises et/ou prêt d'argent) }\end{array}$ & 19 \\
\hline Achats de barriques & 8 \\
\hline $\begin{array}{l}\text { Procurations données à des tiers } \\
\text { pour recouvrer des créances }\end{array}$ & 4 \\
\hline Transactions commerciales et financières diverses & 5 \\
\hline Transactions immobilières & 2 \\
\hline Total & 95 \\
\hline
\end{tabular}

Annexe 2 - Affrètements et connaissements passés par Thomas de Bondié

\begin{tabular}{|c|c|c|c|}
\hline & & pastel & blé \\
\hline \multirow[t]{9}{*}{1505} & avril & 2 & \\
\hline & mai & 2 & \\
\hline & juin & 3 & \\
\hline & juillet & & \\
\hline & août & & \\
\hline & septembre & & \\
\hline & octobre & 9 & \\
\hline & novembre & 11 & \\
\hline & décembre & 3 & \\
\hline \multirow[t]{11}{*}{1506} & janvier & & \\
\hline & février & 3 & \\
\hline & mars & 5 & 1 \\
\hline & avril & 4 & \\
\hline & mai & 3 & \\
\hline & juin & 1 & 3 \\
\hline & juillet & 2 & 1 \\
\hline & août & & \\
\hline & septembre & & \\
\hline & octobre & & \\
\hline & novembre & 3 & \\
\hline
\end{tabular}




\section{Annexe 3}

\section{Actes pour Thomas de Bondié}

11 avril 1505 : reconnaissance de dette (RDD) - Bordeaux/Londres - chanvre dû à P. del Poyo

25 avril 1505 : Connaissement (C) - pastel pour Londres + pastel et vin de T. de Bondié libres de fret

25 avril 1505 : prix-fait - maison

30 avril $1505: \mathrm{C}$ - pastel pour Londres

30 avril 1505 : accord pour arrêter un procès pour dette due à P. del Poyo

7 mai 1507 : RDD - Bordeaux - avoine vendue par P. del Poyo

7 mai 1505 : livraison de barriques - Bordeaux

20 mai 1505 : C - pastel pour Londres

23 mai 1505 : C - pastel pour Arnemuiden

29 mai 1505 : RDD - Bordeaux - chanvre vendu par P. del Poyo

29 mai 1505 : livraison de barriques - Bordeaux

5 juin 1505 : C - pastel pour Bilbao - inspection du navire par T. de Bondié 6 juin 1505 : $\mathrm{C}$ - pastel pour Arnemuiden

9 juin 1505 : vente à réméré d'une maison à Bordeaux en faveur de P. del Poyo/accord avec T. de Bondié

23 juin 1505 : C - pastel pour Londres

9 septembre 1505 : RDD - Soussan - vente de barriques par T. de Bondié

14 octobre 1505 : C - pastel pour Arnemuiden

14 octobre 1505 : C - pastel pour Londres

24 octobre $1505: \mathrm{C}$ - pastel pour Londres

27 octobre 1505 : Quittance - T. de Bondié intermédiaire entre un marchand de Rouen et J. de Bernuy

30 octobre 1505 - C - pastel pour Londres

30 octobre $1505-\mathrm{C}$ - pastel pour Londres

31 octobre $1505-\mathrm{C}$ - pastel pour Londres

31 octobre $1505-\mathrm{C}$ - pastel pour Arnemuiden

31 octobre 1505 - C - pastel pour Arnemuiden

31 octobre 1505 - C - pastel pour Arnemuiden

5 novembre 1505 - Procuration de $\mathrm{T}$. de Bondié à Etienne Ulmyères pour recouvrer de l'argent qui lui est dû

5 novembre 1505 : C - pastel pour Londres - 4 balles à T. de Bondié quittes de fret

6 novembre $1505: \mathrm{C}$ - pastel pour Londres

6 novembre $1505: \mathrm{C}$ - pastel pour Londres

6 novembre $1505: \mathrm{C}$ - pastel pour Londres

6 novembre $1505: \mathrm{C}$ - pastel pour Londres

8 novembre 1505 : C - pastel pour Londres - 10 balles à T. de Bondié quittes de fret

8 novembre $1505: \mathrm{C}$ - pastel pour Londres

10 novembre 1505 : C - pastel pour Arnemuiden 
10 novembre 1505 : C - pastel pour Londres - 10 balles à T. de Bondié quittes de fret

11 novembre 1505 : C - pastel pour Londres - 20 balles et 1 tonneau à T. de Bondié quittes de fret

17 novembre 1505 : C - pastel pour Arnemuiden

3 décembre 1505 : $\mathrm{C}$ - pastel pour Londres - 10 balles pour T. de Bondié quittes de fret

4 décembre 1505 : C - pastel pour Londres - tonneau de vin à T. de Bondié quitte de fret?

16 décembre 1505 : $\mathrm{C}$ - pastel pour Arnemuiden

20 décembre 1505 : RDD pour T. de Bondié par un marchand de Bordeaux suite à la vente de pastel + garantie

24 décembre 1505 : T. de Bondié reconnaît avoir reçu de Coppin de Valladolid 11 lasts de harengs qu'il a vendus. Avec cet argent, il a acheté 15 tx de vin et 16 balles de pastel qui sont chargés sur la Barbe de Penmarch

24 décembre 1505 - RDD - $\mathrm{m}^{\mathrm{e}}$ de navire de Penmarch pour l'achat de 2 tx de vin à T. de Bondié - qu'il reconnaît avoir goûtés

26 décembre 1505 : C - 4 barils d'huile d'olive expédiés par T. de Bondié à Londres - fret à fixer

20 janvier 1506 : Procuration de T. de Bondié à Etienne Ulmyères pour recouvrer de l'argent qui lui est dû

9 février 1506 : RDD - marchands de Bordeaux à T. de Bondié pour l'achat de 5 pipes de pastel + garantie par T. de Bondié sur l'aloi des 5 pipes de pastel

21 février 1506 : C - pastel pour Arnemuiden

26 février 1506 : $\mathrm{C}$ - pastel pour Arnemuiden

27 février 1506 : C - pastel pour Arnemuiden - prêt à la grosse aventure par le $\mathrm{m}^{\mathrm{e}}$ à $\mathrm{T}$. de Bondié

2 mars 1506 : C - pastel pour Arnemuiden

5 mars 1506 : Af : pour porter du blé à Saint-Sébastien ou Pasajes

5 mars 1506 : C - pastel pour Londres

5 mars 1506 : C - pastel pour Londres + 2 barriques de vin à T. de Bondié quittes de fret

20 mars 1506 : C - pastel pour Londres + 1 barrique de vin à T. de Bondié quitte de fret

30 mars 1506 : C - pastel pour Arnemuiden + 1 bar. de vin pour T. de Bondié quitte de fret

7 avril 1506 : Procuration de T. de Bondié à un marchand de Bordeaux pour recouvrer une créance sur un marchand de Montauban suite à la vente de merlus + produit de la vente de 10 pipes de merlus envoyées à un marchand de Toulouse

9 avril 1506 : C - pastel pour Arnemuiden

11 avril $1506: \mathrm{C}$ - pastel pour Londres +1 barrique de vin à T. de Bondié quitte de fret 
15 avril 1506 : Procuration de T. de Bondié à un espagnol pour récupérer une créance sur un marchand navarrais demeurant à La Rochelle ou à La Châtaigneraie en Poitou

21 avril 1506 : C - vin et pastel pour Laredo pour le compte de T. de Bondié +14 pièces de cuir quittes de fret

21 avril 1506 : livraison de barriques à T. de Bondié

21 avril 1506 : livraison de barriques à T. de Bondié

21 avril 1506 : livraison de barriques à T. de Bondié

21 avril 1506 : livraison de barriques à T. de Bondié

24 avril 1506 : Affrètement (Af) - pastel pour Arnemuiden

12 mai 1506 : C - pastel pour Bilbao + 2 douzaines et demie de planches de chêne et une demi-barrique de vinaigre à $\mathrm{T}$. de Bondié quittes de fret

12 mai 1506 : Af - pastel pour Bilbao - avance faite par T. de Bondié + C - le 14 mai

11 mai 1506 : livraison de barriques à T. de Bondié

15 mai 1506 : livraison de barriques à T. de Bondié

21 mai 1505 : attestation par T. de Bondié d'un chargement de pastel pour un marchand de Rouen à destination de Rouen ou de Dieppe + C du 25 mai

25 mai 1506 : C - pastel pour Arnemuiden - prêt de 6 l. 10 s. t. à la grosse aventure du $\mathrm{m}^{\mathrm{e}}$ à $\mathrm{T}$. de Bondié

26 mai 1506 : RDD - Bordeaux - à T. de Bondié

3 juin 1506 : Af - blé pour Lisbonne ou Setubal + C du 25 juin 1506

9 juin 1506 : Af - blé pour Lisbonne ou Setubal + C du 25 juin 1506

13 juin 1506 : C - pastel pour Arnemuiden

23 juin : Af - blé pour Lisbonne ou Setubal (acte inachevé) + C du 25 juin

$1^{\text {er }}$ juillet $1506: \mathrm{C}$ - pastel pour Arnemuiden

7 juillet 1506 : T. de Bondié déclare avoir reçu une lettre de Denis de Belbesère, de Toulouse, mentionnant l'envoi d'une lettre de change de 1000 livres par l'entremise de Jean de Bernuy - faute d'avoir reçu cette somme, T. de Bondié a dû laisser appareiller les navires affrétés - il doit procéder à d'autres affrètement et acheter du blé à plus haut prix/copie d'une lettre de Salvat de la Het en castillan, donnant l'ordre de payer à T. de Bondié les 1000 l. que lui doit un certain Corroal

13 juillet 1506 : C - pastel, vin et vinaigre pour Londres

7 juillet 1506 : suite au contrat passé à Bruges le 13 juin 1506, portant sur la vente par Jean Bisine, md de Bordeaux, à Pierre Lopes, marchand de Calatayud, de 2400 boisseaux de blé pour le prix de 200 écus - quittance de Jean de Bisine suite au paiement des 200 écus

7 juillet 1506 : suite au contrat passé à Bruges le 6 juin 1506, portant sur la vente par Jean Bisine, marchand de Bordeaux, à Diego de Castro, marchand espagnol, de 800 de blé de Zwin -Jean de Bisine constitue son frère Pierre comme garant pour effectuer le chargement

17 juillet 1506 : C - blé pour Lisbonne ou Setubal - faisant suite à un Af du 18 juin 
5 août 1506 : quittance de Jean de Bisine à T. de Bondié pour le paiement de 100 écus en relation avec un chargement de blé pour Pierre de Lopes/ si les 200 pipes n'ont pas été chargées le 12 août, Jean de Bisine doit rembourser les 100 écus - marchand de Bordeaux garant de la transaction

19 août 1506 : RDD - Bordeaux - prêt d'argent (400 l. t. à Pierre de Bisine, marchand de Marmande)

11 septembre 1506 : RDD - vente de barriques à Bordeaux

15 septembre 1506 : RDD - vente de barriques

15 septembre 1506 : RDD - vente de barriques

15 septembre 1506 : RDD - vente de barriques et prêt d'argent - Bordeaux

16 septembre 1506 : RDD - vente de barriques - Bordeaux

16 septembre 1506 : RDD - vente de barriques - Bordeaux

5 septembre 1506 : RDD - vente de barriques - Bordeaux

23 septembre 1506 : RDD - vente de barriques - Bordeaux

30 septembre 1506 : RDD - vente de blé - marchand de Saragosse

14 novembre 1506 : C - pastel pour Arnemuiden

16 novembre : $\mathrm{C}$ - pastel pour Arnemuiden

19 novembre 1506 : Af - pastel (inachevé)

\section{Actes pour Louis de Bondié}

12 avril 1505 : RDD - Bordeaux - pastel vendu par Louis de Bondié + garantie

9 décembre 1506 : $\mathrm{C}-60$ tx de vin à Louis de Bondié pour Arnemuiden ou L'Écluse - prêt à la grosse aventure de 100 l. par le mè̀ L. de Bondié 


\section{RÉSUMÉ}

Les activités commerciales de Thomas de Bondié, bourgeois et marchand de Bordeaux, sont éclairées par une centaine d'actes notariés concentrés entre avril 1505 et décembre 1506. Tout en développant ses propres affaires, il affrète les navires sur lesquels le pastel d'importants marchands de Toulouse est expédié vers l'Angleterre et la Flandre. Lorsque l'occasion se présente Thomas de Bondié n'hésite pas à se lancer dans des opérations sortant de l'ordinaire, tant par les produits traités que par les routes commerciales empruntées. Pour ce marchand, comme pour beaucoup d'autres de son époque, la pluralité des activités constitue une forme de réactivité commerciale et d'adaptation aux opportunités du marché.

\section{ABSTRACT}

The commercial activities of Thomas Bondie, a burgher and merchant of Bordeaux, are known thanks to over a hundred notarial deeds dated between April 1505 and December 1506. While developing his own business, he chartered vessels that shipped pastel to England and Flanders for important merchants of Toulouse. When the opportunity presented itself, Thomas de Bondie did not hesitate to explore new ventures with different products or unfamiliar trade routes. For this merchant, as for many others of his time, pluriactivity was a form of commercial responsiveness that enabled him to adapt to market changes. 